\title{
COMMENTARY
}

\section{Seniors Health Strategic Clinical Network: Age proofing Alberta through innovation}

\author{
Anna Millar PhD, Heather M. Hanson PhD, Adrian Wagg MB; for the Seniors Health Strategic Clinical Network
}

- Cite as: CMAJ 2019 December 4;191(Suppl 1):S19-21. doi: 10.1503/cmaj.190580

B y 2046, 1 in 5 Albertans will be 65 years of age or older, and those aged 80 years and older are expected to double within the same time frame. ${ }^{1}$ Health and social care systems worldwide face considerable challenges to meet the societal and economic demands as more people live longer with multiple comorbidities. ${ }^{2}$ Dementia is a leading cause of disability and dependency among seniors: in 2016, about 42000 Albertans were living with the disease, a number projected to triple by 2046. The cost of dementia is not solely health-related: in 2008, total health and societal (e.g., caregiver-related) costs in Alberta exceeded $\$ 1.2$ billion. ${ }^{3}$

The Seniors Health Strategic Clinical Network's (SH SCN; www.ahs.ca/seniorshealthscn) mission is "to make improvements to healthcare services and practices that enable Alberta's seniors to optimize their health, well-being and independence" (see figure). The SH SCN is a network of health care providers, researchers, policy-makers and patient and family advisors (lay people with lived experiences). It comprises a core committee supported by a small leadership team. The core committee is made up of about 40 individuals including physicians, directors of operations, analysts and patient and family advisors, among others, who meet quarterly. The SH SCN also engages a provincial community of practice with a membership of almost 400 people representing a broad mix of clinicians working in seniors' health and a research network comprising about 100 academics.

The Scientific Office of the SH SCN builds partnerships with researchers to advance the use of knowledge to improve care and health outcomes for seniors. This office also brings members of the research network together to foster collaborative opportunities and multidisciplinary approaches to improving care for seniors, building research capability in Alberta's future workforce through studentships and seed grants. The James Lind Alliance Priority Setting Partnership project has engaged patients, caregivers, seniors' organizations and clinicians to identify 10 provincial priority research questions for seniors' health. ${ }^{4}$

The SH SCN develops and influences research and innovation under a 3-year strategic framework, known as the Transformational Roadmap ${ }^{5}$ (https://www.albertahealthservices.ca/assets/ about/scn/ahs-scn-srs-roadmap.pdf). The roadmap is grounded

\section{KEY POINTS}

- The Seniors Health Strategic Clinical Network's (SH SCN) work is aligned with 3 strategic priorities as outlined in the Transformational Roadmap: Aging Brain Care, Late-life Transitions Initiative (FRAILTI) and Anticipating an Aging Alberta.

- The SH SCN facilitates the flow of information and ideas between research and practice communities to identify and fill knowledge and practice gaps rapidly.

- Work spearheaded by the SH SCN includes provincial initiatives across all care sectors (acute, continuing and community care) including the Appropriate Use of Antipsychotics project and Elder Friendly Care.

by 3 strategic priorities that were identified by and reaffirmed in updates through collaborative engagement with the core committee, patient and family advisors and the communities of practice and research, taking into consideration the current state, planning assumptions and trends to identify opportunities for innovation in Alberta: Aging Brain Care; Frailty, Resilience, Agingwell: Late-life Transitions Initiative (FRAILTI); and Anticipating an Aging Alberta.

Examples of work led by the SH SCN include the Appropriate Use of Antipsychotics project, Elder Friendly Care in Acute Care and the Primary Health Care Integrated Geriatric Services Initiative.

The Appropriate Use of Antipsychotics project involved care teams working collaboratively with residents living in long-termcare facilities and their families to enhance person-centred care by consideration of each resident's life story and recognition of underlying causes of agitation to address their unmet needs. Since this project was implemented in 2014/15, use of antipsychotics in Alberta's long-term-care facilities decreased by more than $30 \%$. At present, $17.1 \%$ of residents in long-term care in Alberta (who do not have a chronic mental health condition) are prescribed antipsychotics compared with the national average of $21.2 \% .{ }^{6}$ Qualitative evaluation provided rich descriptions of individuals "waking up" and becoming more interactive with their families and surroundings again (Appendix 1, available at www.cmaj.ca/lookup/ suppl/doi:10.1503/cmaj.190580/-/DC1). The implementation of 


\section{Transformational Roadmap Summary 2017-2020}
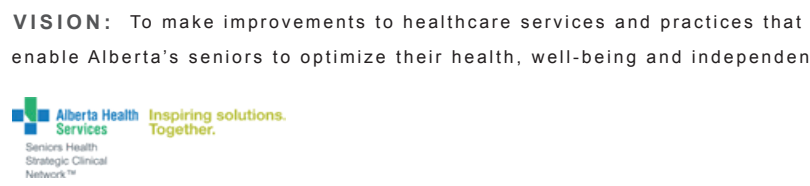

ACTIONS

Care in the Community
Primary Health Care Integrated
Geriatric Service Initiative (PHC IGSI)
Community Grants for innovations in
dementia care

Appropriate Prescribing

for Seniors

Appropriate Use of Anti-psychotics (AUA)

in Long Term Care (LTC) - sustainablity

AUA in Supportive Living

Appropriate Prescribing \& Medication Use

Strategy Among Older Albertans

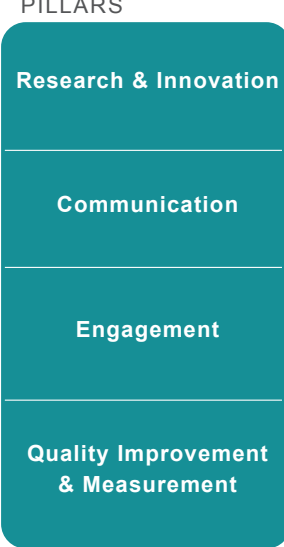

Elder Friendly Care

(EFC) in Acute Care

Acute Care - Provincial

Provincial EFC Strategy

Provincial EFC (Delirium) Toolkit

Alberta Dementia Strategy and

Action Plan (ADSAP)

Alberta Dementia Research Strategy

Community Grants to support

innovations in dementia care

Pain and Depressive Mood Quality

Improvement Project
Anticipating an

Aging Alberta
PROGRESS

AUA in LTC (170 facilities)

$17 \%$ of Alberta's long term care residents

(2017) use antipsychotic medications

$21.7 \%$ national average

A decrease from $28 \%$ to $21 \%$ (from 2012 )

AUA in Supportive Living (179 facilities Useage decrease: $20 \%$ to $17 \%$ (2017)

Acute Care - Provincial

12 acute care sites implemented strategies to

12 acute care sites implemented s
enhance inpatient senior care

Acute Care - EFC (Delirium) Toolkit

Resources developed to support engagement

of acute care sites [across Alberta]

5 PRIHS Grants awarded

Provincial EFC Strategy in development

Alberta Dementia Research Strategy

6 Primary Care Networks and 9 communities

engage with PHC IGSI

Dementia resources for healthcare

providers \& public

Dementia Advice Line and online resources

for public and clinicians

8 Community Innovation Grants for

People Impacted by Dementia

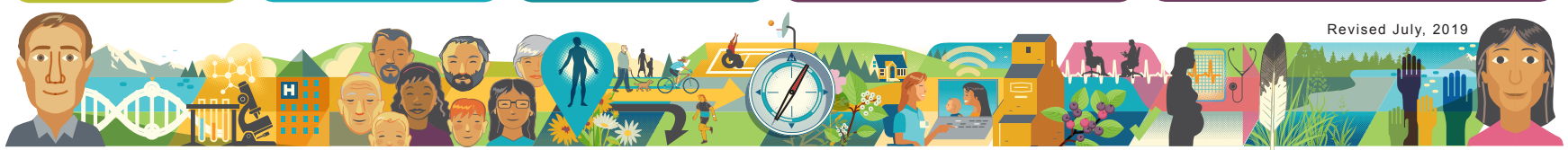

Summary of the Seniors Health Strategic Clinical Network (SH SCN) Transformational Roadmap.

this initiative and its associated resources (including the peerreviewed Choosing Wisely Canada toolkit “When Psychosis Isn't the Diagnosis"7) has gained Alberta recognition as a national leader in appropriate antipsychotic prescribing practices.

The Elder Friendly Care project supports restraint as a last resort, delirium prevention, promotion of continence and functional capacity, and timely discharge of medically complex older adults from the hospital setting. ${ }^{8}$ A study examining Elder Friendly Care approaches implemented in the surgical setting in Alberta reported a significant decrease in complications and length of stay among seniors after acute abdominal surgery. ${ }^{9}$

The Primary Health Care Integrated Geriatric Services Initiative project is a collaboration between the SH SCN and primary care that is designed to enhance the ability of the primary health care team to diagnose and provide care in the community for people living with various comorbidities, including dementia. As part of this initiative, a series of educational workshops providing practical information on topics ranging from timely recognition of dementia to end-of-life care were created to increase capacity to support people living with dementia and their care partners within primary health care.

The SH SCN is driven by the palpable motivation of its members to improve care for older adults, not only provincially, but for 1 person at a time. An example of this is another SH SCN initiative,
The Dementia Advice Line. This is a telephone service that connects family care partners of people with dementia to the compassionate support of skilled nurses as well as to resources in their communities.

The SH SCN is working to ensure that Alberta is prepared for the effect of an older population through change in practice and generation of new knowledge. A strength of the SCN is its ability to facilitate an accelerated pace of change in knowledge translation. In health systems traditional research may take up to 17 years to translate evidence into practice, ${ }^{10}$ yet, the SH SCN has influenced meaningful change in seniors' health since its inception in 2012. A key learning is that optimizing the care of an aging population does not always require additional resources: the health care system can stop doing some things because evidence of benefit is lacking, or because they are inappropriate or inefficient. The SH SCN has a role in identifying such practices (e.g., inappropriate prescribing) and working on strategies to transition away from them. Work is currently underway to identify a set of Quality Indicators, which will be used to monitor outcomes of interest to the work of the SCN.

Perhaps one of the largest challenges that has faced the $\mathrm{SH}$ SCN has been deciding where best to focus its efforts. Innovation is happening across the province; however, the resources that can be directed into areas of importance to seniors' health 
are finite. Furthermore, Alberta is a geographically large and diverse province, which poses an additional challenge for implementing change.

The initiatives in this article illustrate some of the many outputs of the SH SCN to date. In preparation for the unprecedented demographic shift in Canada's population, the SCN continues to align its strategic priorities with Alberta Health Services' pursuit of a patient-centred, quality health system that is both accessible and sustainable for all Albertans.

\section{References}

1. Office of Statistics and Information - Demography. Alberta population projections, 2018-2046 - Alberta, census divisions and economic regions - data tables. Government of Alberta. Available: https://open.alberta.ca/opendata/ alberta-population-projections-2018-2046-alberta-census-divisions-and-economic -regions-data-tables (accessed 2019 Sept. 19).

2. Marengoni A, Angleman S, Melis R, et al. Aging with multimorbidity: a systematic review of the literature. Ageing Res Rev 2011;10:430-9.

3. Alberta dementia strategy and action plan: progress report. Government of Alberta; 2019. Available: https://open.alberta.ca/dataset/2262e7c9-8b18-4d52 -91b1-fb1ed7761ac1/resource/c188d6d8-62f9-4716-8519-2eb2dfa1e926/download /adsap-progress-report-2019-03-06.pdf (accessed 2019 Sept. 19).

4. Seniors Health Strategic Clinical Network. Seniors health research priority setting partnership. Edmonton: Alberta Health Services; 2019. Available: www. albertahealthservices.ca/scns/Page13481.aspx (accessed 2019 Sept. 19).

5. Seniors Health Strategic Clinical Network. 2017-2020 Transformational Roadmap. Edmonton: Alberta Health Services. Available: www.albertahealthservices .ca/assets/about/scn/ahs-scn-srs-roadmap.pdf (accessed 2019 Sept. 19).

6. Seniors Health Strategic Clinical Network. Appropriate use of antipsychotic medications. Edmonton: Alberta Health Services. Available: www.alberta healthservices.ca/assets/about/scn/ahs-scn-sb-seniors-aua.pdf (accessed 2019 Sept. 19).
7. Toolkit: when psychosis isn't the diagnosis. Choosing Wisely Canada. Available: https://choosingwiselycanada.org/perspective/antipsychotics-toolkit/ (accessed 2019 Sept. 19).

8. Seniors Health Strategic Clinical Network. Elder Friendly Care toolkit. Edmonton: Alberta Health Services. Available: www.albertahealthservices.ca/scns/ Page13345.aspx (accessed 2019 Sept. 19).

9. Li Y, Pederson JL, Churchill TA, et al. Impact of frailty on outcomes after discharge in older surgical patients: a prospective cohort study. CMAJ 2018;190: E184-90.

10. Morris ZS, Wooding S, Grant J. The answer is 17 years, what is the question: understanding time lags in translational research. J R Soc Med 2011;104: 510-20.

Competing interests: Anna Millar, Heather Hanson and Adrian Wagg are employees of Alberta Health Services. No other competing interests were declared.

This article has been peer reviewed.

Affiliations: Alberta Health Services (Millar, Hanson, Wagg); Department of Medicine (Wagg), University of Alberta, Edmonton, Alta.; Cumming School of Medicine (Hanson), University of Calgary, Calgary Alta.

Contributors: Anna Millar wrote the first draft of the manuscript. Heather Hanson and Adrian Wagg assisted with drafting the manuscript. All of the authors contributed substantially to the conception and design of the work, reviewed the manuscript critically for important intellectual content, gave final approval of the version to be published and agreed to be accountable for all aspects of the work.

Correspondence to: Anna Millar, anna.millar2@ahs.ca 